\title{
GIANT INSULINOMA IN A 20 YEAR OLD WOMAN WITH BEHAVIOURAL CHANGES FOR 3 YEARS
}

Ekanayake DMNTK ${ }^{1}$, Wanigasiri $\mathrm{U}^{1}$ Suranjan $\mathrm{M}^{1}$ Dissanayake $\mathrm{K}^{2}$

Department of Radiology, National Hospital of Sri Lanka ${ }^{1}$

Department of Pathology, National Hospital of Sri Lanka ${ }^{2}$

Keywords : Giant Insulinoma, Men syndrome

Corresponding Author:Ekanayake D. M. N. T. K. ${ }^{1}$

Copyright:Ekanayake D. M. N. T. K. ${ }^{1}$

(iD) https://orcid.org/0000-0001-7058-9648

\section{Introduction}

Insulinomas are rare neuroendocrine tumours of the pancreas with a reported incidence of 4 cases per 1 million patient year. Most of them are small $(<2 \mathrm{~cm})$ at the time of diagnosis. Giant insulinomas $(>9 \mathrm{~cm})$ are extremely rare with only less than 50 cases reported in literature up to date. Giant insulinomas are more likely to be malignant than their smaller counter parts. They present with clinical manifestations related to hypoglycemia. However, symptoms maybe vague and nonspecific causing a lag of definitive diagnosis even up to several decades. Radiologist has a key role to play in the diagnosis and management of these patients. Our report highlights a rare case of giant insulinoma detected in a 20 year old female who was investigated for increased sleep and altered behaviour for 3 years' duration.

\section{Case Presentation}

A 20 year old female presented to medical unit with seizures and altered behavior. She had been apparently well until the age of 17 years, after which the family members noticed a gradual decline in her school performance. She had increased daytime sleep $>12$ hours, recurrent episodes of falls, episodes of sleep paralysis, significant weight gain and features of hirsutism. She also had visual and $3^{\text {rd }}$ person auditory hallucinations and generalized seizures. She had been extensively investigated for behavioral changes which comprised of sleep studies, EEG and brain MRI. They all were found to be inconclusive. She was treated subsequently for narcolepsy, migraine and a dissociative disorder and defaulted. She was readmitted to medical unit with seizures and found to have recurrent hypoglycemia which resolved with IV Dextrose administration. She had no family history suggestive of a similar illness or history of Multiple Endocrine Neoplasia.In physical examination she was normotensive, had a BMI of 25 and abdominal examination did not reveal any abdominal mass. Biochemical investigations revealed a fasting glucose level of $14 \mathrm{mg} / \mathrm{dl}$, a high insulin level $23 \mu \mathrm{U} / \mathrm{ml}$ and a C-peptide level of $1.86 \mathrm{ng} / \mathrm{ml}$. Serum calcium, PTH and prolactin levels were within normal limits. A contrast enhanced CT abdomen with a 64 slice scanner and $1 \mathrm{~mm}$ thick slices after administration of $100 \mathrm{ml}$ iodinated contrast was performed at $40 \mathrm{~s}$ arterial phase. It revealed a $9 \mathrm{~cm}(\mathrm{AP}) \mathrm{x} 10.1 \mathrm{~cm}(\mathrm{CC}) \mathrm{x} 8 \mathrm{~cm}(\mathrm{~T})$ sized avidly enhancing mass in the tail of the pancreas closely related to the splenic hilum, greater curvature of the stomach and left kidney. No evidence of adjacent organ invasion was noted. It contained few central calcifications but there were cystic components. The lesion was compressing the splenic artery and vein. There were few varices around the lesion. There was no pancreatic duct dilatation or any other similar lesions elsewhere in the pancreas. MRI abdomen further revealed 2 focal lesions in segment VIII of liver measuring approximately $2 \mathrm{~cm}$ each which were also enhancing avidly in the arterial phase. There were no significant para aortic lymph nodes or peritoneal deposits. Diagnosis of giant insulinoma with liver metastasis was made. 
Patient eventually underwent distal pancreaticosplenectomy along with intraoperative radiofrequency ablation (RFA) for segment VIII liver lesions. Her hypoglycemic state dramatically improved following surgery.

\section{Discussion}

Insulinomas are rare pancreatic neuroendocrine tumours with a reported incidence of only 2 to 3 cases per 1 million patient year. There is a slightly higher prevalence in women compared to men. Patients with insulinomas usually present with the classic triad of symptoms known as Whipple triad. i.e. symptoms of hypoglycemia, fasting hypoglycemia $<50 \mathrm{mg} / \mathrm{dl}$ and relief of symptoms following glucose administration. However, the diagnosis can be delayed from months to several years due to low degree of suspicion given the rarity of the condition. Insulinomas in young patients maybe a part of MEN 1 syndrome.

The risk of malignancy increases with the size of the tumour. Tumours $>3 \mathrm{~cm}$ carry a significant malignancy risk. Insulinomas measuring more than $9 \mathrm{~cm}$ are regarded as giant insulinomas. The WHO 2017 grading for pancreatic neuroendocrine neoplasms (pNENs) take into account histopathological criteria such as mitotic index and Ki 67labeling index to grade pNENs. Additionally, the diagnosis of malignant transformation is based on presence of metastasis to the liver, presence of lymph node deposits and evidence of gross local invasion of adjacent organs on imaging. Even though they are equally distributed in head, body and tail of the pancreas the available literature says that giant insulinomas have a predilection for the tail of the pancreas. As the large majority of pNENs are less than $<2 \mathrm{~cm}$, one of the many diagnostic challenges in insulinomas is preoperative localization and exclusion of the presence of multiple tumours. CT and MRI are essential in this regard with the sensitivity of newer scanners approaching 33\%-64\% and 40\%-90\% respectively. Endoscopic ultrasound too is beneficial in localizing small tumours with sensitivity reaching $86 \%$ to $90 \%$. The appearance of insulinomas on EUS is quite characteristic, with most tumors homogeneously hypoechoic, rounded in shape, and with distinct margins However, the gold standard for localization is intraoperative palpation and intraoperative ultrasound.

Generally insulinomas are avidly enhancing in arterial phase when compared to normal pancreatic parenchyma as all other pNENs. They may contain calcifications. On MRI they appear low signal on T1W and high signal on T2W sequences. Fat suppressed T1W, Gadolinium enhanced and DWI sequences are of use. For confirmation of insulinoma another useful radiological investigation is Ga-68 DOTATE PET-CT. The rationale is, insulinomas express a large number of somatostatin receptors to which Ga-68 DOTATE has an affinity. The sensitivity of this imaging modality is known to be close to 90\%. Unfortunately Ga-68 DOTATE scan was not available in our set up to carry out on our patient. Selective calcium stimulation test with hepatic venous sampling is another radiologically important invasive method of evaluating for occult insulinomas. Surgical resection is curative for insulinomas, with larger lesions requiring more aggressive surgical measures as in our patient. Liver metastasis can be surgically resected or be subjected to RFA/ microwave ablation.

Recurrence is a major concern especially in malignant insulinomas. Therefore, follow up with cross sectional imaging preferably with CT is necessary at regular time intervals.

Reported incidence of recurrence in 10 years after curative resection is less than $6 \%$. 


\section{References}

1. Elizabeth A. Mittendorf, Yao-Chang Liu, Christopher R. McHenry, Giant Insulinoma: Case Report and Review of the Literature, The Journal of Clinical Endocrinology \& Metabolism, Volume 90, Issue 1, 1 January 2005, Pages 575-580, https://doi.org/10.1210/jc.2004-0825

2. Ueda K, Taira T, Hakoda H, et al. Giant insulinoma: report of a case and review of published reports. Surg Case Rep. 2016;2(1):136. doi:10.1186/s40792-016-0265-z

3. Vasikasin V, Watthanatham J, Napatharatip P, Termmathurapoj S. Giant insulinoma in a 15year-old man: A case report. Int J Surg Case Rep. 2016;24:135-138. doi:10.1016/j.ijscr.2016.05.037

4. Okabayashi T, Shima Y, Sumiyoshi T, et al. Diagnosis and management of insulinoma. World J Gastroenterol. 2013;19(6):829-837. doi:10.3748/wjg.v19.i6.829

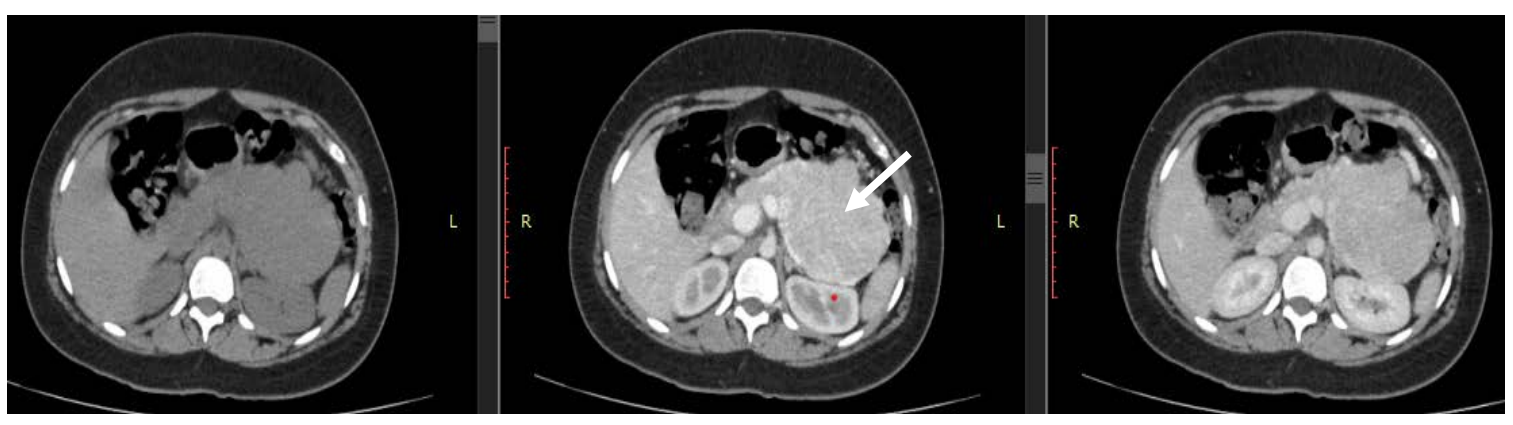

Fig. 1. CECT abdomen arterial phase and portal venous phase images showing giant insulinoma at the tail of the pancreas causing mass effect on splenic vessels and left kidney 


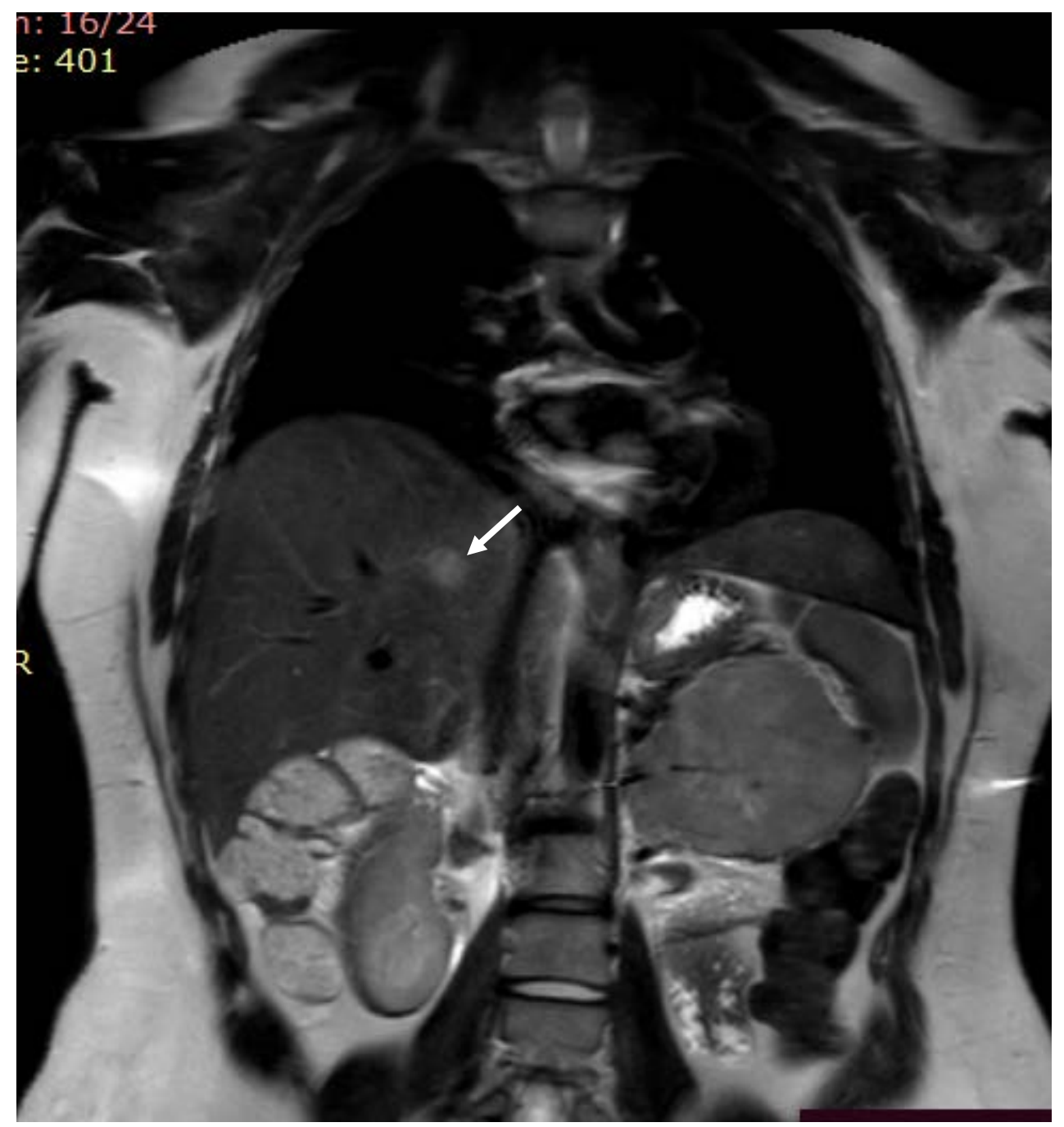

Fig. 2. Coronal T2W MRI image showing segment VIII liver metastasis 